\title{
Cognitive Factors Affecting Free Recall, Cued Recall, and Recognition Tasks in Alzheimer's Disease
}

\author{
Takashi Yamagishi $^{a}$ Takuya Sato $^{\mathrm{b}}$ Atsushi Sato ${ }^{\mathrm{b}}$ \\ Toru Imamuraa, c \\ a Department of Health and Welfare, Graduate School of Health and Welfare, Niigata University \\ of Health and Welfare, and bivision of Speech Therapy, Department of Rehabilitation and \\ 'Department of Neurology, Niigata Rehabilitation Hospital, Niigata, Japan
}

\section{Key Words}

Word recall · Word recognition - Divided attention - Executive functions - Mini-Mental State

Examination • Alzheimer's Disease Assessment Scale • Alzheimer's disease

\begin{abstract}
Background/Aims: Our aim was to identify cognitive factors affecting free recall, cued recall, and recognition tasks in patients with Alzheimer's disease (AD). Subjects: We recruited 349 consecutive AD patients who attended a memory clinic. Methods: Each patient was assessed using the Alzheimer's Disease Assessment Scale (ADAS) and the extended 3-word recall test. In this task, each patient was asked to freely recall 3 previously presented words. If patients could not recall 1 or more of the target words, the examiner cued their recall by providing the category of the target word and then provided a forced-choice recognition of the target word with 2 distracters. The patients were divided into groups according to the results of the free recall, cued recall, and recognition tasks. Multivariate logistic regression analysis for repeated measures was carried out to evaluate the net effects of cognitive factors on the free recall, cued recall, and recognition tasks after controlling for the effects of age and recent memory deficit. Results: Performance on the ADAS Orientation task was found to be related to performance on the free and cued recall tasks, performance on the ADAS Following Commands task was found to be related to performance on the cued recall task, and performance on the ADAS Ideational Praxis task was found to be related to performance on the free recall, cued recall, and recognition tasks. Conclusion: The extended 3-word recall test reflects deficits in a wider range of memory and other cognitive processes, including memory retention after interference, divided attention, and executive functions, compared with word-list recall tasks. The characteristics of the extended 3-word recall test may be advantageous for evaluating patients' memory impairments in daily living.
\end{abstract}

Copyright ๑ 2012 S. Karger AG, Basel 


\section{Introduction}

Deficits of recent memory are one of the major symptoms in many forms of dementia. Evaluating its severity is important in the diagnosis and management of dementia. One of the most commonly used tasks for the cognitive screening of recent memory deficit is the free recall of several words [1]. For example, the Mini-Mental State Examination (MMSE) [2, 3] contains a free recall task of 3 words. This task provides a useful measure for assessing the presence or absence of recent memory deficits but is reported to be insufficient to assess the severity of impairment because of its floor effect $[4,5]$.

In clinical practice, many neurologists, psychiatrists, and clinical neuropsychologists supplement free recall tasks with cued recall tasks that include category cues and forcedchoice recognition tasks for patients who scored zero on a task of free word recall [1].

Various types of cognitive dysfunction may also affect the scores of tasks for recent memory $[6,7]$. However, we do not sufficiently understand cognitive factors other than recent memory that may affect the free recall, cued recall, and recognition tasks of the extended 3-word recall test. This study examined these issues using a statistical model to control for appropriate clinical factors.

\section{Subjects}

We recruited 349 consecutive, community-dwelling patients who attended the Memory Clinic at Niigata Rehabilitation Hospital (Niigata, Japan). The eligibility criterion for the study was a diagnosis of probable AD based on the criteria of the National Institute of Neurological and Communicative Disorders and Stroke and Alzheimer's Disease and Related Disorders Association (NINCDS-ADRDA) [8]. In the initial assessment, each patient underwent an MMSE, an Alzheimer's Disease Assessment Scale (ADAS) [9, 10], a neurological examination, a brain MRI or CT scan, an electroencephalogram, and a blood analysis including vitamin $B_{1}, B_{12}$, and thyroid function. Formal clinical criteria were used to exclude patients with other dementing diseases or delirium.

Table 1 demonstrates the patients' demographic, clinical, and cognitive characteristics.

\section{Methods}

Procedure for the Extended 3-Word Recall Test (Free Recall, Cued Recall, and Recognition Tasks)

Each patient underwent the free recall task from the 3-word recall test within the MMSE. We used 'rabbit' from the animal category, 'airplane' from the vehicle category, and 'tulip' from the flower category as the 3 target words of the recall test. We used these words because of their moderate level of familiarity in Japanese. Words with high familiarity in the three categories, such as 'dog', 'train', and 'cherry blossoms', were avoided as target words in an attempt to reduce the number of incidentally correct responses during the cued recall task.

When a patient could not recall 1 or more of the 3 target words during the free recall task, the examiner immediately introduced the cued recall and recognition tasks. We conducted the cued recall and recognition tasks for each word that a patient failed to recall. The examiner instructed each patient as follows: 'I will give you the category of the target word to help you recall.' The category word for the cue was 'animal' for rabbit, 'vehicle' for airplane, and 'flower' for tulip. If the patient could not recall the target after the presentation of the category word, the examiner introduced a forced-choice recognition trial that con- 
Table 1. Demographic data and scores for all patients and each patient group

\begin{tabular}{|c|c|c|c|c|c|c|c|}
\hline & $\begin{array}{l}\text { All patients } \\
(\mathrm{n}=349)\end{array}$ & $\begin{array}{l}\text { FR1-3 } \\
(n=68)\end{array}$ & $\begin{array}{l}\text { FR0 } \\
(n=281)\end{array}$ & $\begin{array}{l}\text { CR1-3 } \\
(n=101)\end{array}$ & $\begin{array}{l}\text { CR0 } \\
(n=180)\end{array}$ & $\begin{array}{l}\text { RT1-3 } \\
(\mathrm{n}=111)\end{array}$ & $\begin{array}{l}\text { RT0 } \\
(n=69)\end{array}$ \\
\hline emale $g$ & 76.5 & 69.1 & 78.3 & 81.2 & 76.7 & 76.6 & 76.8 \\
\hline Age at examination, years & $79.9 \pm 6.7(58-96)$ & $77.2 \pm 6.8$ & $80.5 \pm 6.6$ & $80.3 \pm 6.5$ & $80.6 \pm 6.6$ & $80.0 \pm$ & $81.5=$ \\
\hline Disease duration, years & $3.4 \pm 2.4(0-15)$ & $3.1 \pm 2.1$ & $3.5 \pm 2.4$ & $3.2 \pm 2.3$ & $3.7 \pm 2.5$ & $3.6 \pm 2.5$ & 4.0 \\
\hline MMSE total score & $18.9 \pm 4.1(5-30)$ & $22.6 \pm 3.6$ & $18.0 \pm 3.7$ & $19.3 \pm 3.3$ & $17.3 \pm 3.8$ & $17.7 \pm 3.9$ & $16.6 \pm 3.5$ \\
\hline ADAS total score ${ }^{1}$ & $19.3 \pm 8.6(4-50)$ & $12.7 \pm 5.7$ & $20.9 \pm 8.4$ & $17.6 \pm 7.4$ & $22.7 \pm 8.4$ & $21.0 \pm 7.7$ & $25.4 \pm 8.7$ \\
\hline Word Recall (0-10) & $6.3 \pm 1.6(0-9)$ & $5.3 \pm 1.5$ & $6.6 \pm 1.5$ & $6.2 \pm 1.4$ & $6.9 \pm 1.5$ & $6.6 \pm 1.5$ & $7.2 \pm 1.4$ \\
\hline Orientation $(0-8)$ & $3.8 \pm 2.2(0-8)$ & $2.1 \pm 2.0$ & $4.2 \pm 2.1$ & $3.6 \pm 2.0$ & $4.5 \pm 2.0$ & $4.3 \pm 2.0$ & $4.8 \pm 2.1$ \\
\hline Following Commands $(0-5)$ & $1.2 \pm 1.0(0-5)$ & $1.0 \pm 0.8$ & $1.2 \pm 1.1$ & $0.9 \pm 0.9$ & $1.4 \pm 1.1$ & $1.3 \pm 1.1$ & $1.5 \pm 1.2$ \\
\hline Ideational Praxis $(0-5)$ & $1.8 \pm 1.7(0-5)$ & $1.1 \pm 1.5$ & $2.0 \pm 1.7$ & $1.6 \pm 1.6$ & $2.3 \pm 1.7$ & $2.0 \pm 1.7$ & $2.8 \pm 1.7$ \\
\hline Naming $(0-5)$ & $0.1 \pm 0.5(0-4)$ & $0.0 \pm 0.1$ & $0.1 \pm 0.5$ & $0.1 \pm 0.2$ & $0.2 \pm 0.6$ & $0.1 \pm 0.4$ & $03 \pm 0.8$ \\
\hline Constructions $(0-5)$ & $1.0 \pm 0.8(0-4)$ & $0.7 \pm 0.8$ & $1.0 \pm 0.8$ & $0.9 \pm 0.9$ & $1.1 \pm 0.8$ & $1.1 \pm 0.9$ & $1.1 \pm 0.7$ \\
\hline
\end{tabular}

Data are shown as the mean $\pm \mathrm{SD}$ (range), unless otherwise indicated. ${ }^{1}$ A higher score indicates greater dysfunction.

Fig. 1. Definition of the patient groups, based on the results of the extended 3-word recall test.

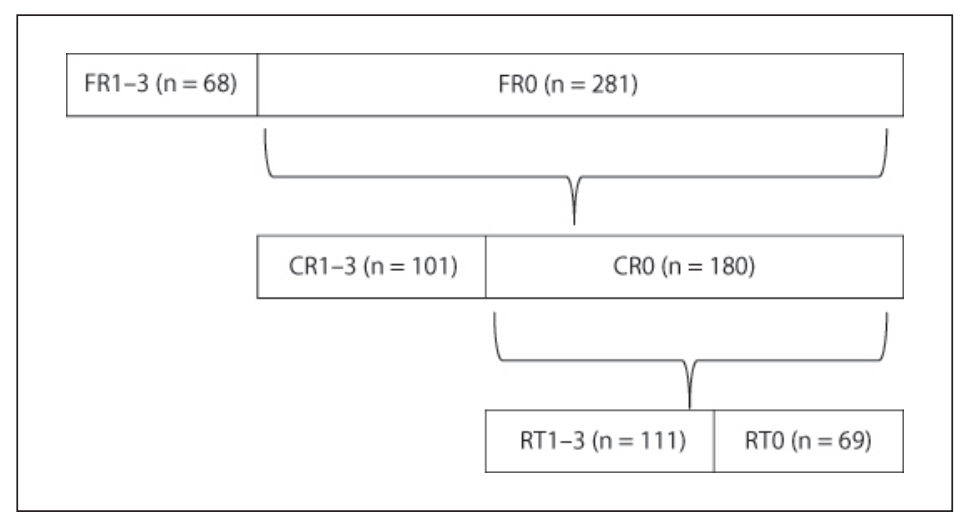

sisted of the target word and 2 distracter words. The 3-word lists for each target were as follows: rat, rabbit, and sheep; bus, airplane, and taxi; and cherry blossoms, sunflower, and tulip.

Definition of the Patient Groups Based on the Results of the Extended 3-Word Recall Test (fig. 1)

Free Recall Group. The patients of the free recall (FR) group were divided into two subgroups: patients who recalled 1 or more words during the free recall task (FR1-3 group) and patients who recalled no words during the free recall task (FR0 group).

Cued Recall Group. The FR0 patients were divided into two subgroups: patients who recalled 1 or more words during the cued recall task (CR1-3 group) and patients who recalled no words during the cued recall task (CR0 group).

Recognition Group. The CR0 patients were further divided into two subgroups: patients who recalled 1 or more words during the recognition task (RT1-3 group) and patients who recalled no words during the recognition task (RT0 group).

Table 1 and figure 1 show the number of patients and their gender distribution, as well as the average age, estimated dementia onset, total MMSE score, total ADAS score, and ADAS subscores for each patient group. 
Statistical Method

Analysis 1

A univariate logistic regression analysis for repeated measures was carried out to evaluate the effects of clinical and cognitive factors on the free recall, cued recall, and recognition tasks in the extended 3-word recall test. The model included the patient groups (FR1-3 vs. FR0, CR1-3 vs. CR0, or RT1-3 vs. RT0) as repeated measures, and one of the patient characteristics (gender, age, or disease duration) or one of the cognitive measures (the scores of the ADAS Word Recall, Orientation, Following Commands, Ideational Praxis, Constructions, or Naming tasks) as an independent variable.

Statistical analyses were conducted using Statistica software (Statsoft Japan, Tokyo, Japan), and significance was defined as $\mathrm{p}<0.05$. We did not correct the significance level for multiple comparisons because of the exploratory nature of this study.

Analysis 2

To control for the effects of age and recent memory deficit, we carried out a multivariate logistic regression analysis for repeated measures. The model included the group as repeated measures, the score on each of 5 ADAS subitems (Orientation, Following Commands, Ideational Praxis, Constructions, and Naming tasks) as an independent variable, and the patients' age and score on the ADAS Word Recall task as confounding factors. This model elucidated the net effect of other cognitive measures after eliminating the effects of the patients' age and any recent memory deficits, represented by the patients' scores on the ADAS Word Recall task.

This study protocol was approved by the Ethics Committee of Niigata University of Health and Welfare.

\section{Results}

Analysis 1

Table 2 shows the results of the univariate logistic regression analysis for each group of patients. Scores on the free recall task (FR1-3 vs. FR0) were significantly related to age and scores on the ADAS Word Recall, Orientation, Ideational Praxis, and Constructions tasks. In addition, there was a nonsignificant trend toward group differences on the ADAS Following Commands and Naming tasks $(\mathrm{p}<0.10)$.

Scores on the cued recall task (CR1-3 vs. CR0) were significantly related to scores on the ADAS Word Recall, Orientation, Following Commands, Ideational Praxis, and Constructions tasks. In addition, there was a nonsignificant trend toward group differences on the ADAS Naming task $(\mathrm{p}<0.10)$.

Scores on the recognition task (RT1-3 vs. RT0) were significantly related to scores on the ADAS Word Recall and Ideational Praxis tasks.

Analysis 2

Table 2 shows the results of the multivariate logistic regression analysis for each patient group. Scores on the free recall task (FR1-3 vs. FR0) were significantly related to scores on the ADAS Orientation task and exhibited a trend toward correlation with scores on the ADAS Ideational Praxis task. That is, patients with more errors on the ADAS Orientation and Ideational Praxis tasks more frequently scored zero on the free recall task.

Scores on the cued recall task (CR1-3 vs. CR0) were significantly related to scores on the ADAS Orientation, Following Commands, and Ideational Praxis tasks. That is, patient with more errors on the ADAS Orientation, Following Commands, and Ideational Praxis tasks more frequently scored zero on the cued recall task. 
Tasks in Alzheimer's Disease

Table 2. The results of each regression analysis for each patient group

\begin{tabular}{|c|c|c|c|c|c|c|c|c|c|}
\hline & \multicolumn{3}{|c|}{ FR1-3 versus FR0 } & \multicolumn{3}{|c|}{ CR1-3 versus CR 0} & \multicolumn{3}{|c|}{ RT1-3 versus RT0 } \\
\hline & $\begin{array}{l}\text { odds } \\
\text { ratio }\end{array}$ & $95 \% \mathrm{CI}$ & $\mathrm{p}$ value & $\begin{array}{l}\text { odds } \\
\text { ratio }\end{array}$ & $95 \%$ CI & $\mathrm{p}$ value & $\begin{array}{l}\text { odds } \\
\text { ratio }\end{array}$ & $95 \% \mathrm{CI}$ & $\mathrm{p}$ value \\
\hline \multicolumn{10}{|l|}{ Univariate logistic regression analysis } \\
\hline Age at examination (years) & 0.93 & $0.89-0.97$ & $<0.01$ & 0.99 & $0.96-1.03$ & 0.69 & 0.97 & $0.92-1.01$ & 0.15 \\
\hline Gender & 1.6 & $0.83-3.13$ & 0.16 & 0.76 & $0.40-1.45$ & 0.41 & 1.01 & $0.27-3.81$ & 0.98 \\
\hline Disease duration (years) & 0.91 & $0.80-1.03$ & 0.15 & 0.91 & $0.81-1.01$ & 0.09 & 0.93 & $0.82-1.05$ & 0.24 \\
\hline \multicolumn{10}{|l|}{ ADAS } \\
\hline Word Recall (0-10) & 0.55 & $0.45-0.67$ & $<0.01$ & 0.71 & $0.60-0.85$ & $<0.01$ & 0.73 & $0.59-0.92$ & $<0.01$ \\
\hline Orientation $(0-8)$ & 0.60 & $0.51-0.70$ & $<0.01$ & 0.80 & $0.71-0.91$ & $<0.01$ & 0.90 & $0.77-1.04$ & 0.16 \\
\hline Following Commands $(0-5)$ & 0.77 & $0.58-1.02$ & $<0.10$ & 0.64 & $0.49-0.83$ & $<0.01$ & 0.88 & $0.70-1.15$ & 0.34 \\
\hline Ideational Praxis $(0-5)$ & 0.70 & $0.57-0.84$ & $<0.01$ & 0.78 & $0.67-0.91$ & $<0.01$ & 0.76 & $0.63-0.91$ & $<0.01$ \\
\hline Constructions $(0-5)$ & 0.59 & $040-0.85$ & $<0.01$ & 0.72 & $0.53-0.98$ & $<0.05$ & 0.95 & $0.66-1.36$ & 0.77 \\
\hline Naming $(0-5)$ & 0.17 & $0.02-1.19$ & $<0.10$ & 0.47 & $0.22-1.02$ & $<0.10$ & 0.73 & $0.44-1.21$ & 0.21 \\
\hline \multicolumn{10}{|c|}{$\begin{array}{l}\text { Multivariate logistic regression analysis with age and ADAS Word Recall score as confounding factors } \\
\text { ADAS }\end{array}$} \\
\hline Orientation $(0-8)$ & 0.67 & $0.56-0.80$ & $<0.01$ & 0.86 & $0.75-0.98$ & $<0.05$ & 0.97 & $0.82-1.14$ & 0.70 \\
\hline Following Commands $(0-5)$ & 1.02 & $0.75-1.40$ & 0.88 & 0.72 & $0.55-0.94$ & $<0.05$ & 0.97 & $0.72-1.30$ & 0.84 \\
\hline Ideational Praxis $(0-5)$ & 0.83 & $0.68-1.02$ & $<0.10$ & 0.84 & $0.72-0.99$ & $<0.05$ & 0.80 & $0.66-0.97$ & $<0.05$ \\
\hline Constructions $(0-5)$ & 0.79 & $0.53-1.19$ & 0.26 & 0.83 & $0.60-1.15$ & 0.26 & 1.08 & $0.74-1.59$ & 0.68 \\
\hline Naming $(0-5)$ & 0.35 & $0.06-2.02$ & 0.24 & 0.62 & $0.29-1.32$ & 0.21 & 0.80 & $0.46-1.38$ & 0.41 \\
\hline \multicolumn{10}{|c|}{$\begin{array}{l}\text { Stepwise multiple logistic regression analysis with age and ADAS Word Recall score as confounding factors } \\
\text { ADAS }\end{array}$} \\
\hline Orientation $(0-8)$ & 0.67 & $0.56-0.79$ & $<0.01$ & & & & & & \\
\hline Following Commands (0-5) & & & & 0.72 & $0.55-0.94$ & $<0.05$ & & & \\
\hline Ideational Praxis $(0-5)$ & & & & & & & 0.80 & $0.66-0.97$ & $<0.05$ \\
\hline \multicolumn{10}{|l|}{ Constructions $(0-5)$} \\
\hline Naming $(0-5)$ & & & & & & & & & \\
\hline
\end{tabular}

Scores on the recognition task (RT1-3 vs. RT0) were significantly related to scores on the ADAS Ideational Praxis task. That is, patients with more errors on the ADAS Ideational Praxis task more frequently scored zero on the recognition task.

We additionally conducted a stepwise multiple logistic regression analysis for repeated measures. The model included the group as repeated measures, the patients' age and score on the ADAS Word Recall task as confounding factors, and the patients' scores on the significant cognitive measures in Analysis 2 as an independent variable. Table 2 shows the results of the stepwise multiple logistic regression analysis for each patient group. The significant variables were scores on the ADAS Orientation task for groups FR1-3 and FR0, scores on the ADAS Following Commands task for groups CR1-3 and CR0, and scores on the ADAS Ideational Praxis task for groups RT1-3 and RT0.

\section{Discussion}

Free recall, cued recall with category cues, and forced-choice recognition tasks of word recall are classic clinical tools in behavioral neurology, neuropsychiatry, and clinical neuropsychology [1]. In this study, we confirmed a significant relation between scores of the ADAS Word Recall test, a standard neuropsychological test of recent memory, and the results of the 
free recall, cued recall, and recognition tasks of the extended 3-word recall test. These findings demonstrate the validity of these common clinical tasks.

We also found that scores on the free recall, cued recall, and recognition tasks were significantly related to scores on several cognitive ADAS tasks after we eliminated the effects of the patients' ages and recent memory deficits, as represented by scores on the ADAS Word Recall task. Tasks with correlated results included the ADAS Orientation task and the free and cued recall tasks, the ADAS Following Commands task and the cued recall task, and the ADAS Ideational Praxis task and the free recall, cued recall, and recognition tasks. We discuss the potential underlying mechanisms of these relationships below.

\section{Relationship between Scores on the ADAS Orientation Task and the Free Recall} and Cued Recall Tasks

We hypothesize that the relationship between the results of the ADAS Orientation task and the free recall and cued recall tasks reflects a common deficit in a subprocess of recent memory, namely, retention after interference. In Analysis 2, the statistical model controlled for the effects of recent memory deficits, as represented by scores on the ADAS Word Recall task. In this task, subjects are asked to recall the 10 target words immediately after their presentation, but subjects are not asked to retain the target words after other tasks. Therefore, the statistical method of this study may not sufficiently control for the effect of impaired retention after interference. In contrast, the results of both the free and cued recall tasks of the extended 3-word recall test depend on the subjects' retention of the target words after interference tasks. The ADAS Orientation task scores the subjects' responses to 8 questions about the year, month, date, day, time, season, place, and their person. Patients with preserved retention after interference are thought to score well because they may recall current episodes, which help them correctly orient to time, place, and person. Thus, decreased scores on the ADAS Orientation task and failures on the free and cued recall tasks may result from a common deficit in memory retention after interference.

Relationship between Scores on the ADAS Following Commands and Cued Recall Tasks

We hypothesize that the relationship between the results of the ADAS Following Commands task and the cued recall task reflects a disturbance of divided attention. Divided attention refers to an individual's ability to simultaneously respond to multiple demands [1]. The ADAS Following Commands task evaluates motor responses to simple and complex verbal commands. Therefore, this task measures attentional functionality in addition to language comprehension. A correct response to a complex command necessitates divided attention.

Why does cued recall suffer when the patients' ability to divide their attention is impaired? The category cue of the cued recall task in this study was presented to patients immediately after failure of free recall for each target word. Processes of successful retrieval in cued recall tasks are thought to be as follows. During the free recall, the recall of the target words fails if the target word was insufficiently activated and the degree of activation was below the threshold of retrieval and recall. The categorical cue then activates the target and related words. If the patient has sufficient divided attention, the summed activation from the below-threshold activation of the target word during the free recall and the activation of the target word after presentation of the categorical cue reaches the threshold of retrieval and recall. On the other hand, if patients have a deficit of divided attention, they do not retain the activation from the free recall, and the target word fails to be retrieved and recalled during the cued recall. Thus, decreased scores on the ADAS Following Commands task and failures on the cued recall task may result from a common deficit in divided attention. 
Relationship between the ADAS Ideational Praxis Task and Free Recall, Cued Recall, and Recognition Tasks

We hypothesize that the relationship between the results of the ADAS Ideational Praxis task and the free recall, cued recall, and recognition tasks reflects a disturbance of executive functioning. Executive functioning is intrinsic to the ability to respond in an adaptive manner to novel situations and is the basis of many cognitive and social skills. Deficits in executive functioning, including organization, strategy, and self-monitoring, can affect memory performance and other cognitive tasks $[1,7]$. The ADAS Ideational Praxis task requires the execution of a complex sequence of motor actions involving the use of 6 tools and objects. Executive functioning is required to use the items in the appropriate sequence [11].

The free recall, cued recall, and recognition tasks also become difficult when executive functioning is impaired. In the encoding process of the extended 3-word recall test, executive functioning provides cognitive strategies for finding effective cues, such as a visual image for or the semantic category of each word. These cues can facilitate the recall of target words during the free recall, cued recall, and recognition tasks. Thus, patients with disturbances of executive functioning have difficulty performing the ADAS Ideational Praxis task and employing effective strategies to encode and retrieve the target words during the extended 3-word recall test.

\section{Conclusion}

The results of this study suggest that scores on the extended 3-word recall test (with free recall, cued recall, and recognition tasks) reflect deficits in a wider range of memory and other cognitive processes than do word-list recall tasks. Processes that may be probed by this test include memory retention after interference, divided attention, and executive functioning. The characteristic of the extended 3-word recall test may be advantageous for predicting memory impairments in daily living because the above-mentioned deficits in the cognitive processes may also affect everyday memory, that is, memory in natural contexts in the real world [12]. A validated scoring system for the extended 3-word recall test may provide a valid measure for predicting the presence and severity of memory impairments in daily living. We believe this issue is worthy of future clinical research.

\section{Acknowledgement}

The authors wish to express their appreciation to the staff of Niigata Rehabilitation Hospital for help with data collection.

\section{Disclosure Statement}

The authors declare no conflicts of interest. 


\section{References}

1 Lezak MD, Howieson DB, Loring DW, et al: Neuropsychological Assessment, ed 4. New York, Oxford University Press, 2004.

-2 Folstein MF, Folstein SE, McHugh PR: 'Mini-Mental State': A practical method for grading the cognitive state of patients for the clinician. J Psychiatr Res 1975;12:189-198.

3 Mori E, Mitani Y, Yamadori A: Usefulness of a Japanese version of the Mini-Mental State Test in neurological patients. Jpn J Neuropsychol 1985;1:82-90.

4 Kawahata N, Gotoh C, Yokoyama S: Neuropsychological differentiation between dementia of Alzheimer type and vascular dementia using mini-mental state examination (MMSE). Jpn J Neuropsychol 2001;17:223-229.

5 Honda T, Ito N, Sato A, Imamura T: Addition of the cued recall and recognition tasks to Mini Mental State Examination distinguishes aged normal subjects from Alzheimer's disease with mild memory impairment. Jpn J Neuropsychol 2006;22:233-239.

6 Wilson BA: Case Studies in Neuropsychological Rehabilitation. New York, Oxford University Press, 1999.

7 Walsh KW: Understanding Brain Damage. A Primer of Neuropsychological Evaluation, ed 2. London, Churchill Livingstone, 1991.

-8 Mckhann G, Drachman D, Folstein M, et al: Clinical diagnosis of Alzheimer's disease: report of the NINCDS-ADRDA Work Group under the auspices of Department of Health and Human Service Task Force on Alzheimer's Disease. Neurology 1984;34:939-944.

-9 Mohs RC, Rosen WG, Davis KL: The Alzheimer's Disease Assessment Scale: an instrument for assessing treatment efficacy. Psychopharmacol Bull 1983;19:448-450.

10 Homma A, Fukuzawa K, Tsukada Y Ishii T, Hasegawa K: Development of a Japanese version of Alzheimer's Disease Assessment Scale (ADAS). Jpn J Geriatr Psychiatry 1992;3:647-655.

11 Oikawa N, Oguri R, Sato A, Imamura T: Executive dysfunction on activity of daily living (ADL) in Alzheimer's disease: a study using Dysexecutive Questionnaire (DEX). Jpn J Neuropsychol 2006;22: $138-145$.

12 Cohen G: Memory in the Real World. New Jersey, Lawrence Erlbaum Associates, 1989. 